\title{
4 Müssen wir unserem Gefühl folgen? Ansätze des Sentimentalismus
}

\author{
4.1 Philosophische Grundlagen \\ 4.2 Humes Tugendlehre \\ 4.3 Diskussion
}

Nachleben eines Monsters: Hobbes' düsteres Menschenbild (siehe Kap.3.2), seine Angriffe auf die weltlichen Machtansprüche von Glaubensgemeinschaften und seine süffisant-trockene Kritik jeder Berufung auf die Offenbarung wirken noch lange nach seinem Tod skandalös. Mit Hobbes' Verteidigung einer absolutistischen Monarchie sind nicht einmal die Monarchen selbst zufrieden, die ihre Autorität lieber dem persönlichen Gottesgnadentum als den niederen Sicherheitsbedürfnissen ihrer Untertanen verdanken wollten - Bedürfnissen, die durch jeden beliebigen Emporkömmling oder Eroberer ebenso gut befriedigt werden könnten, sofern er denn zur Herrschaft gelangt. Zur Widerlegung der hobbesschen Ideen werden hunderte von Büchern geschrieben. Der den Staat im Bild eines biblischen Monsters erfassen wollte, gilt nun selbst als das Monster of Malmesbury. Obwohl man sich kaum offen auf ihn berufen kann, bleiben Hobbes' Überlegungen jedoch einflussreich. Zu gut passen sie zu den die Neuzeit bestimmenden neuen Überzeugungen und Haltungen. Hobbes' Einfluss ist auch in der Moralphilosophie David Humes (17111776) unverkennbar. Zugleich ist Humes Ethik jedoch auch von der Moral-sense-Tradition des schottischen Sentimentalismus geprägt, einer Schule der Moralphilosophie, die von einem weit freundlicheren Menschenbild ausgeht und prosozialen Gefühlen, Wohlwollen und Empathievermögen, eine zentrale Rolle zuweist. Humes Ethik kombiniert den interessensbasierten vertragstheoretischen Ansatz, wie er von Hobbes vertreten wurde, mit einer Gefühlstheorie der Moral. Sie tut dies auf eine Weise, die durch ihre radikalisierte Grundlagenkritik die ethische Diskussion nachhaltig prägen wird.

\subsection{Philosophische Grundlagen}

Empiristische Erkenntnistheorie: Im ersten Buch seines Hauptwerks A Treatise of Human Nature untersucht Hume das Erkenntnisvermögen, im zweiten die menschlichen Leidenschaften einschließlich der Motivations- und Handlungstheorie und im dritten die Moralphilosophie. Auf dem Gebiet der Erkenntnistheorie vertritt Hume einen Empirismus, der Annahmen Lockes radikal weiterdenkt. Der gesamte Gehalt unseres Bewusstseins besteht aus Wahrnehmungen (perceptions), die sich in Eindrücke (impressions) und Ideen (ideas) unterscheiden lassen. Die Ideen

Impressions and ideas
Kontraktualismus und Sentimentalismus 
Kausalitätsproblem sind blassere Abbilder der Eindrücke oder, im Fall komplexer Ideen, aus solchen zusammengesetzt. Ideen, die nicht auf Eindrücke zurückgehen - seingeborene Ideen ‘, wie sie von Vertretern des Rationalismus angenommen werden - gibt es nicht. Dem konsequenten Empiristen Hume bleibt auch die Tatsache nicht verborgen, dass wir Kausalität als solche niemals wahrnehmen können. Was wir wahrnehmen, ist beispielsweise nur, dass eine Billardkugel eine andere trifft und diese sich zu bewegen beginnt. Die von uns unterstellte kausale Kraft, als deren Ergebnis wir dieses Phänomen begreifen, sehen wir jedoch nicht; sie ist kein möglicher Gegenstand der Wahrnehmung. Hume zufolge haben Kausalaussagen daher ihre Basis nur in der psychologischen Gewohnheit, Ereignisse, die regelmäßig in bestimmter Reihenfolge nacheinander auftreten, als verbunden $\mathrm{zu}$ interpretieren.

\section{Hume lesen}

Hume legt seine philosophischen Überzeugungen in systematischer und detaillierter Form in dem 1738-1740 erschienenen Werk A Treatise of Human Nature dar (Hume 2007). Unzufrieden mit der zögerlichen Rezeption dieses Werks präsentiert er dessen zentrale Ideen in etwas vereinfachter Form erneut in seinen zwei Büchern An Enquiry Concerning $\mathrm{Hu}$ man Understanding (1748) und An Enquiry Concerning the Principles of Morals (1751). In der zweiten, moralphilosophischen Enquiry fehlen einige Argumente (etwa für die These der Passivität der Vernunft; s. u.), die Hume möglicherweise für zu voraussetzungsvoll für ein breiteres Publikum gehalten hat. Umgekehrt finden sich alle wesentlichen Thesen und fast alle Argumente der Enquiry bereits im Treatise. Die maßgebliche Ausgabe der humeschen Werke ist die kritische und kommentierte Clarendon Hume Edition Series, nach der im Folgenden zitiert wird. Konventionsgemäß werden vor den Seitenzahlen der zitierten Ausgabe noch die jeweils vorhandenen Gliederungsebenen (Teil, Kapitel, Abschnitt und Absatz) angegeben. Als Oxford Philosophical Texts (OPT) stehen teils textidentische Studienausgaben der Werke zur Verfügung (der Text des Treatise in der OPT-Ausgabe ist ab deren 11. Auflage mit dem Text der kritischen Ausgabe identisch). Ältere, von Selby-Bigge editierte Ausgaben sind urheberrechtsfrei im elektronischen Faksimile verfügbar. Deutschsprachige Übersetzungen von Humes Werk sind nur mit Vorsicht zu gebrauchen.

Rationalität und Wahrheit: Vernunft versteht Hume als das Vermögen der Beurteilung wahrheitsfähiger Annahmen. Wahrheit und Falschheit kann nur von Behauptungen über Relationen ausgesagt werden, die entweder das Verhältnis von Ideen untereinander betreffen - etwa das Verhältnis zwischen den Ideen `Junggeselle`, `Mann`, und sunverheiratet ‘ oder die Übereinstimmung zwischen Ideenverbindungen und gemeinsam auftretenden Eindrücken ausdrücken - etwa die Übereinstimmung zwischen der Ideenrelation, dass die Marsoberfläche rot ist und dem Eindruck einer rötlichen Färbung beim Blick auf die Marsoberfläche. Humes 
Interpretation von Wahrheit und Vernunft - wenn man so will: seine deskriptivistische Rationalitätstheorie - spielt eine wichtige Rolle in seinem Argument gegen einen ethischen Rationalismus.

"Desires" als Motivationsgrundlage: Im zweiten Teil des Treatise entwickelt Hume die Grundlagen einer Motivations- und Handlungstheorie, die in weiterentwickelten Formen die Diskussion bis heute prägt (vgl. v. a. Davidson 2001, insbes. Essay 1; Williams 1981, Kap. 8; zur Übersicht Stout 2005; als radikale Kritik vgl. Bittner 2005). Grundlage der Handlungsmotivation ist Hume zufolge ein a-rationaler innerer Zustand, den er als desire, affection oder passion bezeichnet, was mit "Verlangen«, »Leidenschaft« oder »Neigung« nur unvollkommen übersetzt werden kann. Gemeint ist eine wie auch immer gefärbte, grundsätzlich positive Haltung gegenüber einem bestimmten Weltzustand (entsprechend spricht Davidson später vorsichtig, aber umständlich von einer »Pro-Einstellung«; vgl. Davidson 2001). A-rational ist der mentale Zustand eines desires, weil - Rationalität sich lediglich auf die Untersuchung von Wahrheit oder Falschheit bezieht,

- Wahrheit oder Falschheit nur von Behauptungen ausgesagt werden kann, die auf Relationen bezogen sind,

- ein desire keine Relation ist, sondern ein einfacher innerer Zustand.

Selbstverständlich kann zwar die Behauptung, dass jemand ein bestimmtes Verlangen hat, wahr oder falsch sein, denn damit wird ja das Vorliegen einer Relation behauptet (nämlich zwischen der betreffenden Person und ihrem Verlangen). Das Verlangen selbst aber ist als solches nicht wahrheitsfähig und insofern auch kein Gegenstand einer rationalen Beurteilung oder Kritik. Der Unterschied zwischen beliefs und desires wird gegenwärtig in einer der Sprechakttheorie entstammenden Metaphorik häufig mit ihrer gegensätzlichen >Passungsrichtung` (direction of fit) erläutert. Demnach stellen beliefs geistige Zustände dar, die an der Welt ausgerichtet werden sollen (mind-to-world direction of fit), während umgekehrt desires darauf zielen, die Welt an den desires auszurichten (worldto-mind direction of fit; u. a. Anscombe 1957; Smith 1994, S. $111 \mathrm{ff}$.; dazu kritisch Copp/Sobel 2001; Jacobson-Horowitz 2006).

Vernunft und Handlungsmotivation: Freilich können desires erst dann eine Handlung motivieren, wenn bestimmte Überzeugungen (beliefs) hinzutreten (das ist die Annahme, die Davidson zum sog. Belief-desireModell der Handlungsmotivation ausgearbeitet hat). Mein Verlangen, ein Glas Gin zu trinken (meine positive Einstellung gegenüber einem Weltzustand, in dem ich Gin trinke) wird mich nur dann zu der Handlung motivieren, den Inhalt des vor mir stehenden Glases zu leeren, wenn ich überzeugt bin, dass dieses Glas Gin enthält und dass ich, wenn ich es leere, den gewünschten Weltzustand herbeiführe. Insofern solche Überzeugungen kritisierbar sind, sind, wie Hume ausführt, auch Handlungen bzw. Handlungsmotive rationaler Beurteilung zugänglich: Gegenstand wahrheitsfähiger Behauptungen können zum einen Aussagen über die Existenz von erstrebten Objekten meines Verlangens sein (in dem Glas dort befindet sich der von mir erstrebte Gin - oder nein, es ist Benzin) und zum anderen Aussagen über die Effektivität und Effizienz von Mit-
Motivation basiert auf a-rationalen Verlangen

Belief-desireModell 
Hume 2007, 3.1.1.12, S. 295

Vernunft als

Sklavin der Leidenschaften

Hume 2007, 2.3.3.4, S. 266

Hume 2007, 2.3.3.6, S. 267 teln zur Realisierung des erstrebten Weltzustandes (nein, Gin lässt sich nicht aus Benzin destillieren). Entsprechend kann Vernunft unsere Handlungen nur auf zweierlei Weise beeinflussen:

"It has been observ'd, that reason, in a strict and philosophical sense, can have an influence on our conduct only after two ways: Either when it excites a passion by informing us of the existence of something which is a proper object of it; or when it discovers the connexion of causes and effects, so as to afford us means of exerting any passion."

Es ist wichtig, diese Passage genau zu lesen. Hume will mit der ersten Möglichkeit keineswegs sagen, dass die Vernunft eine Leidenschaft oder Neigung produziert oder sins Dasein ruft . Es ist vielmehr so, dass die Vernunft, indem sie uns über die Existenz eines Objekts einer Leidenschaft informiert, diese latent - als Gefühlsdisposition - bereits vorhandene Leidenschaft erregt oder anfacht (excites). Mit der zweiten Möglichkeit wiederum ist lediglich gemeint, dass die Vernunft uns Mittel und Wege aufzeigt, unsere Leidenschaft oder unseren Affekt auszuleben (to exert). Keineswegs will Hume hier sagen, dass die Vernunft "uns die Mittel [zeigt], irgendeinen Affekt zu erzeugen «, wie eine deutsche Übersetzung sinnentstellend vorschlägt (Hume 2007b, S. 18; Hervorh. M. W.).

Passivität der Vernunft: Der Punkt ist bedeutsam, weil er eine zentrale und folgenreiche These Humes betrifft: Vernunft ist steril, sie kann allein, d.h. ohne die Mitwirkung eines bereits existierenden Verlangens, kein neues Verlangen produzieren. Weil ohne ein solches Verlangen keine Handlungsmotivation zustande kommt (die dem Belief-desire-Modell zufolge immer beide Elemente benötigt) kann Vernunft allein Handlungen oder Affekte weder verhindern noch hervorbringen: "reason is perfectly inert, and can never either prevent or produce any action or affection" (Hume 2007, 3.1.1.8, S. 294). Da die Rolle der Vernunft in praktischen Zusammenhängen auf die Suche nach möglichen Objekten und geeigneten Mitteln zur Befriedigung bereits existierender Leidenschaften beschränkt ist, kommt ihr lediglich eine dienende Funktion zu. Ohne Furcht vor Zuspitzungen bezeichnet Hume in einer berühmten Passage des Treatise die Vernunft als Sklavin der Leidenschaften:

"Reason is, and ought only to be the slave of the passions, and can never pretend to any other office than to serve and obey them."

Handlungspräferenzen sind demnach auch nicht rationaler Kritik zugänglich, wie Hume an einem ebenso prägnanten Beispiel illustriert:

"Where a passion is neither founded on false suppositions, nor chooses means insufficient for the end, the understanding can neither justify nor condemn it. 'Tis not contrary to reason to prefer the destruction of the whole world to the scratching of my finger."

Handlungen mögen lobenswert oder tadelnswert sein, sie können aber nicht vernünftig oder unvernünftig sein (ebd., 3.1.1.10, S. 295). Prakti- 
sche Vernunft ist bestenfalls als instrumentelle Vernunft $\mathrm{zu}$ verstehen. Tatsächlich ist nicht völlig klar, ob Hume ein Vertreter eines Konzepts instrumenteller Vernunft oder vielmehr ein Vernunftskeptiker ist. Ein Konzept instrumenteller Vernunft zu vertreten bedeutet $\mathrm{zu}$ behaupten, dass Vernunft uns niemals letzte Ziele vorschreiben oder empfehlen kann, dass sie uns jedoch eine rationale Verpflichtung zur Wahl eines Mittels auferlegt, soweit wir uns an ein Ziel gebunden wissen: Wer den Zweck will, muss auch die Mittel wollen. Ein Vernunftskeptiker will der Vernunft nicht einmal diese eingeschränkte Orientierungsfunktion zuschreiben. Dass Hume ein Vernunftskeptiker sein könnte, wird durch eine eigentümliche Passage nahegelegt, in der er behauptet, es sei nicht einmal gegen die Vernunft, das von uns selbst für geringer gehaltene Gut einem größeren Gut vorzuziehen (ebd., 2.3.3.6, S. 267). Die Passage ist jedoch nicht eindeutig. Insgesamt spricht mehr dafür, dass Hume eine Konzeption instrumenteller Vernunft vertritt (Railton 2006).

Ruhige und leidenschaftliche Affekte: Vernunft und Leidenschaften können sich demnach auch nicht im Widerstreit befinden, da die Vernunft überhaupt keine Kraft hat, die sie dem Verlangen entgegensetzen könnte. Wie ist dann jedoch zu erklären, dass ein solcher Widerstreit zwischen Vernunft und Leidenschaft immer wieder angenommen wird? Wie sind die traditionellen ethischen Ermahnungen zu interpretieren, wir sollten unsere Leidenschaften der Vernunft unterwerfen - man denke etwa an Platons Allegorie des Wagenlenkers, der zufolge der vernünftige Seelenteil die triebhaften und leidenschaftlichen Seelenteile zügeln und lenken soll? Solche Ermahnungen sind nach Humes Auffassung tatsächlich sinnlos. Sie erklären sich aus einer zwar psychologisch nachvollziehbaren, aber gleichwohl gravierenden Verwechslung. Nicht alle Neigungen und Affekte präsentieren sich nämlich in derselben Weise. Es gibt unter ihnen solche, die die Seele gewaltig aufwühlen (violent emotions; ebd., 2.2.3.9, S. 268), und solche, die so ruhig und beständig wirken, dass sie sich der Wahrnehmung entziehen und als unsichtbarer Unterstrom unseres Gefühlslebens nur an ihren Wirkungen erkennbar sind (calm desires and tendencies). Die ruhigen Verlangen sind von zweierlei Art. Es handelt sich einerseits um natürliche und instinktive Anlagen wie Wohlwollen (benevolence) oder moralischen Groll (resentment), die Liebe zum Leben oder die Freundlichkeit gegenüber Kindern. Diese Art der calm passions wirft keine Probleme auf und spielt als Grundlage der Humeschen Moraltheorie eine klare Rolle. Eine zweite (und im Rahmen seines Systems problematischere) Art ruhigen Verlangens sieht Hume in dem "general appetite to good, and aversion to evil, consider'd merely as such « (ebd., 2.2.3.8 f., S. 268). Aufgrund dieses ruhigen und unauffälligen Charakters einiger Leidenschaften wird ihr Einfluss Hume zufolge immer wieder mit dem Einfluss der Vernunft verwechselt. Was man als Willensstärke preist, ist aber in Wahrheit nicht die Herrschaft der Vernunft über die Leidenschaften, sondern ein Charakter, in dem die ruhigen Leidenschaften stärker ausgeprägt sind als die gewaltsamen.

Moral und Motivation: Vernunft allein hat also keinen Einfluss auf unsere Handlungen und Leidenschaften. Moral hingegen hat einen solchen Einfluss: Hume zufolge zeigt uns die Erfahrung, dass Menschen 
häufig durch ihr Pflichtgefühl zu Handlungen motiviert oder durch ihre Einschätzung, dass bestimmte Handlungen ungerecht sind, von deren Ausführung abgehalten werden (ebd., 3.1.1.5, S. 294). Er vertritt damit eine Auffassung, die als metaethischer Internalismus bezeichnet wird.

Metaethischer Internalismus bezeichnet die Auffassung, wonach moralische Überzeugungen oder Haltungen als solche motivational bedeutsam sind. Metaethische Internalisten nehmen an, dass das Sich-zueigen-Machen eines moralischen Prinzips notwendigerweise mit einer motivationalen Disposition einhergeht, gemäß diesem Prinzip zu handeln - so schwach diese Disposition auch sein und so häufig sie in der Konkurrenz mit anderen Neigungen auch unterliegen mag. Der internalistischen Auffassung zufolge wäre es inkonsistent, zugleich zu behaupten: "Peter ist überzeugt, moralisch verpflichtet zu sein, pünktlich zur Verabredung zu kommen" und zu behaupten: "Peter hat keinerlei Motiv, pünktlich zur Verabredung zu kommen«. Metaethische Externalisten bestreiten einen entsprechenden Zusammenhang. (Diese Verwendung der Begriffe "Externalismus" und "Internalismus" ist von ihrem Gebrauch im Rahmen der philosophischen Erkenntnistheorie zu unterscheiden.)

Anti-Rationalismus: Aus dem zuvor Gesagten folgert Hume, dass der ethische Rationalismus, wie er beispielsweise von dem eine Generation älteren Samuel Clarke vertreten wurde, unhaltbar ist. Denn da Moral einen Einfluss auf unsere Handlungen und Effekte hat, während Vernunft allein einen solchen Einfluss unmöglich ausüben kann, folgt notwendigerweise, dass Moral nicht allein der Vernunft entstammt. Hume hat dem ethischen Rationalismus damit die Grundlage entzogen. Moralische Urteile können, anders als Clarke behauptet, keine Vernunftwahrheiten ausdrücken.

\section{Humes Argument gegen den moraltheoretischen Rationalismus}

Humes Argument für die These, dass Moral nicht allein der Vernunft entstammen kann, lässt sich lose als eine Sequenz zweier Syllogismen verstehen, wobei die Konklusion des ersten Syllogismus eine Prämisse des zweiten Syllogismus bildet.

Prämisse 1: Belief-desire-Modell der Handlungsmotivation Überzeugungen (beliefs) können nur gemeinsam mit Verlangen (desires) Handlungen motivieren.

\section{Prämisse 2: Deskriptivistische Rationalitätstheorie}

Vernunft kann nur Überzeugungen begründen, nicht aber Verlangen erzeugen oder aus der Welt schaffen.

\section{$\rightarrow$ Konklusion 1: Passivität der Vernunft}

Vernunft allein kann keine Handlungen motivieren. 
Prämisse 3: Metaethischer Internalismus

Moral motiviert Handlungen.

Konklusion 2: Moraltheoretischer Anti-Rationalismus

Moral kann nicht (allein) der Vernunft entstammen.
Sein-Sollens-Fehlschluss: In einer einflussreichen, aber nicht sehr klaren und unterschiedlich interpretierten Passage im moralphilosophischen Teil des Treatise wendet sich Hume gegen seiner Auffassung nach fehlerhafte Argumentationen in allen moralphilosophischen Texten, die ihm bislang untergekommen sind:

"In every system of morality, which I have hitherto met with, I have always remark'd, that the author proceeds for some time in the ordinary way of reasoning, and establishes the being of a God, or makes observations concerning human affairs; when of a sudden I am surpriz'd to find, that instead of the usual copulations of propositions, is, and is not, I meet with no proposition that is not connected with an ought, or an ought not. This change is imperceptible; but is, however, of the last consequence. For as this ought, or ought not, expresses some new relation or affirmation, 'tis necessary that it shou'd be observ'd and explain'd; and at the same time that a reason shou'd be given, for what seems altogether inconceivable, how this new relation can be a deduction from others, which are entirely different from it."

Wenn man `Seinsaussagen ‘ als Feststellungen versteht, wonach etwas der Fall ist, und 'Sollensaussagen ‘ als Feststellungen, die besagen, dass etwas der Fall sein oder etwas getan werden soll, vertritt Hume also die These, dass aus `Seinsaussagen ‘ nicht ohne weitere Erläuterung und Begründung sSollensaussagen abgeleitet werden können. Das mag zunächst trivial scheinen. Zweifellos ist es nicht ohne Veränderung des Wahrheitswertes einer Aussage möglich, ein »ist" durch ein »soll« zu ersetzen. Dass ein Stuhl grün ist impliziert nicht, dass dieser Stuhl grün sein soll. Unter bestimmten Interpretationen des Wortes »sein" mag schon diese Behauptung nicht trivial wirken. Wie erwähnt, versteht der auf Platon zurückgehende metaphysische Naturalismus "Sein« als graduierbaren Wesens- und Wertbegriff. In diesem Rahmen mögen ganz ähnliche Aussagen wie die über den grünen Stuhl durchaus plausibel scheinen: Der Mensch ist vernünftig, darum soll der Mensch auch vernünftig sein. Die Rede von »dem Menschen « ist dabei freilich mehrdeutig: In der Seinsaussage bezeichnet »der Mensch« das Wesen bzw. die Idee des Menschen, die immer nur mehr oder weniger realisiert ist, in der Sollensaussage ist hingegen mit »der Mensch" der reale Mensch gemeint, der offenbar seinem Ideal noch nicht vollständig entspricht. Die Sollensaussage, dass reale Menschen die Idee des Menschen realisieren sollen, wird also implizit schon als Prämisse vorausgesetzt, und Humes Überlegung lässt sich in diesem Zusammenhang als Aufforderung verstehen, diese unausgesprochene Prämisse explizit zu machen. Im Rahmen des Empirismus,
Hume 2007,

3.1.1.27, S. 302

Differenz zwischen Sein und Sollen 
wie er von Hume vertreten wird, wird Platons Zusatzprämisse freilich unverständlich, denn `Ideen treten dort nur noch als schwacher Nachklang von Eindrücken auf und nicht als Ideale, denen wir nachstreben sollten.

Hume wendet sich jedoch nicht nur gegen die Vorstellung, dass eine vereinzelte Seinsaussage unmittelbar in eine Sollensaussage umgewandelt werden kann. Er behauptet überdies, dass aus einer Menge von Seinsaussagen ohne zusätzliche Begründung keinerlei Sollensaussage abgeleitet werden kann. Was bedeutet diese Annahme, die manchmal auch als Humes Gesetz oder als Sein-Sollen-Dichotomie bezeichnet wird? Hume gibt an dieser Stelle keine weiteren Erläuterungen. Seine Intentionen bezüglich dieser Passage können daher nur aus dem Kontext seiner moralphilosophischen Überzeugungen erschlossen, deren metaethische Interpretation allerdings ebenfalls kontrovers ist. Entsprechend besteht kein Konsens über die Frage, unter welcher Interpretation die Passage einen guten Sinn ergibt.

Lesarten der humeschen Distinktion: Die Lesart, die der Passage die radikalste Aussage zuordnet, sieht darin ein Bekenntnis zum Nonkognitivismus.

Eine grundlegende Kontroverse der Metaethik betrifft die Frage, ob moralische Urteile (z. B.: "Es ist verwerflich, zu lügen«) einen Wahrheitswert haben, d. h. ob sie überhaupt wahr oder falsch sein können. Kognitivisten gehen von der Wahrheitsfähigkeit moralischer Urteile aus. Nonkognitivisten nehmen hingegen an, dass moralische Urteile entgegen dem Eindruck, der durch ihre sprachliche Form erzeugt wird, weder wahr noch falsch sein können (siehe Kap. 8).

Sie nimmt an, dass Hume jede Art der Gewinnung von Sollensaussagen aus Seinsaussagen für unmöglich erklären wollte. Diese Auffassung habe Hume jedoch nur vertreten können, wenn er überzeugt gewesen sei, dass Werturteile nicht als wahrheitsfähige Tatsachenaussagen interpretiert werden können. Am anderen Ende des Spektrums steht die Interpretation, dass Hume lediglich eine logische Ableitbarkeit von Seinsaussagen aus Sollensaussagen bestreiten wollte. Auch der Schluss von »A ist ein Quadrat" auf »A ist ein Rechteck" (Schluss $Q-R$ ) ist freilich kein logisch gültiger Schluss. Logisch gültig sind Schlüsse nur dann, wenn die Wahrheit der Schlussfolgerung schon aufgrund ihrer Form zwingend aus der Wahrheit der Prämissen folgt. $Q-R$ ist aber nur aufgrund des spezifischen Inhalts der verwandten Begriffe »Quadrat « und »Rechteck« wahr. Um Q-R in einen logisch gültigen Schluss umzuwandeln, wäre die Einführung eines Brückenprinzips nötig (»Alle Quadrate sind Rechtecke«), das als zweite Prämisse fungiert.

Entsprechend könnte es sich (so diese vorsichtige Lesart der Passage) bei dem zusätzlichen Grund, den Hume für einen legitimen Übergang von Seinsaussagen zu Sollensaussagen für nötig gehalten habe (»a reason shou'd be given«, s. o.), um Brückenprinzipien handeln, die den implizi- 
ten Sollens- oder Empfehlungscharakter bestimmter Seinsaussagen explizit machen. Beispielsweise würde der Schluss von "Handlung $H$ ist grausam« auf »Man soll $H$ unterlassen« das Brückenprinzip erfordern »Man soll grausame Handlungen unterlassen«. Dieser Deutung zufolge würde Hume lediglich einen Spezialfall des allgemeinen logischen Grundsatzes bekräftigen, dass die Konklusion eines gültigen Schlusses nichts Substantielles enthalten kann, dass nicht auch schon in den Prämissen enthalten war. Humes Gesetz hätte dann die begrenzte Funktion, diejenigen, die moralische Auffassungen zu begründen versuchen, zu zwingen, alle Prämissen explizit zu machen. Die entscheidende metaethische Frage, inwieweit bestimmte Arten von Seinsaussagen tatsächlich normative Gehalte haben, die dann durch angemessene Brückenprinzipien zum Vorschein gebracht werden können, wäre damit aber noch nicht beantwortet. Vertreter/innen bestimmter Spielarten des moralischen Realismus könnten beispielsweise weiterhin die Auffassung vertreten, dass die Aussage: »Man soll nichts Grausames tun" ähnlich selbstverständlich aus unserem Verständnis von Grausamkeit und von Sollen folgt wie die Aussage: "Alle Quadrate sind Rechtecke" aus unserem Verständnis von Quadraten und Rechtecken (z. B. Audi 2006, S. 2; Foot 2001, S. 78; Ross 2009, S. 137).

Anti-Naturalismus: Auch wenn seine Überlegungen zum Sein-SollensFehlschluss damit vereinbar sein mögen, teilt Hume selbst diese metaethische Auffassung allerdings nicht. Er wendet sich explizit nicht nur gegen einen moralischen Rationalismus, der moralische Prinzipien allein aus »Ideen« logisch deduzieren will, sondern auch gegen einen moralischen Naturalismus, dem zufolge moralische Eigenschaften (die Tugendhaftigkeit oder Lasterhaftigkeit einer Handlung oder eines Charakters) natürliche Tatsachen oder feststellbare Relationen sind. Betrachte man allein die objektiven Aspekte einer Handlung, beispielsweise eines Mordes, werde ihr moralischer Charakter völlig unsichtbar:

"Take any action allow'd to be vicious: Wilful murder, for instance. Examine it in all lights, and see if you can find that matter of fact, or real existence, which you call vice. In which-ever way you take it, you find only certain passions, motives, volitions and thoughts. There is no other matter of fact in the case."

Moralische Brückenprinzipien

Hume 2007, 3.1.1.26, S. 301

\section{2 | Humes Tugendlehre}

Gefühlstheorie der Moral: Der überwiegende Teil der dargestellten Überlegungen war bislang kritischer Art. Hume hat der Vernunft auf dem Gebiet der Praxis eine bestenfalls instrumentelle Rolle als "Sklavin der Leidenschaften« zugewiesen. Er hat den ethischen Rationalismus und Realismus zurückgewiesen. Er hat die Ableitung von Sollensaussagen aus Seinsaussagen kritisiert. Auf welcher Grundlage lässt sich vor diesem Hintergrund noch eine Moralphilosophie errichten? Die Antwort gibt Hume direkt im Anschluss an das zuletzt angeführte Zitat: Der moralische Charakter, etwa die Verwerflichkeit eines Mordes, entgeht einem nur so lange, 
Hume 2007, 3.1.1.26, S. 301

Moralische Qualitäten als Gegenstand der Gefühlswahrnehmungen

Moralische Bedeutung des Charakters "till you turn your reflexion into your own breast, and find a sentiment of disapprobation, which arises in you, towards this action. Here is a matter of fact; but 'tis the object of feeling, not of reason. It lies in yourself, not in the object. So that when you pronounce any action or character to be vicious, you mean nothing, but that from the constitution of your nature you have a feeling or sentiment of blame from the contemplation of it. Vice and virtue, therefore, may be compar'd to sounds, colours, heat and cold, which, according to modern philosophy, are not qualities in objects, but perceptions in the mind [...]."

Humes Antwort liegt also wiederum in den Gefühlen. Die wesentliche Basis moralischer Urteile liegt sin unserer eigenen Brust ; in unseren Gefühlen als Betrachter/innen moralisch relevanter Geschehnisse. Mit dieser Auffassung reiht Hume sich in die Tradition der Moral-Sense-Theorie bzw. des Sentimentalismus ein, zu der Autoren wie Shaftesbury, Butler, Hutcheson und auch der etwas jüngere, heute vornehmlich als Ökonom bekannte Adam Smith gehören. Hume vergleicht moralische Qualitäten mit sekundären Qualitäten wie Farbe oder Kälte, die lediglich subjektiver Natur seien. Er knüpft hier an die unter anderem von Locke betonte Unterscheidung zwischen primären Qualitäten und sekundären Qualitäten an. Primäre Qualitäten wie Masse oder Ausdehnung werden dabei als objektive Eigenschaften der Dinge verstanden, während die durch sekundäre Qualitäten präsentierten Phänomene (etwa der Farbwahrnehmung oder des Geschmacks) nur durch die spezifische Art unserer subjektiven Sinnesausstattung zu erklären sind (dazu Kemmerling 2007; siehe auch Kap. 8.4.3).

Hume als Tugendethiker: Gegenstand der ethischen Beurteilung sind im Rahmen der humeschen Ethik allerdings nicht so sehr Handlungen oder Handlungsnormen, als vielmehr Charakterzüge. Der primäre ethische Wertbegriff der humeschen Ethik ist dementsprechend nicht der Begriff der Richtigkeit, sondern der Tugend. Wenigstens in diesem formalen Sinn ist Hume zweifellos ein Vertreter der Tugendethik (zur weiteren Diskussion vgl. Swanton 2007). Die moralische Beurteilung von Handlungen ist gegenüber der Beurteilung des Charakters in systematischer Hinsicht nachrangig: "[t]he external performance has no merit« (Hume 2007, 3.2.1.2, S.307). Wir billigen oder missbilligen Handlungen nur in Abhängigkeit von unserer Billigung oder Missbilligung der Charakterzüge, die sich in ihnen manifestieren. Hinsichtlich unserer Erkenntnismöglichkeiten müssen wir bei der moralischen Beurteilung des Charakters freilich wiederum von dem Eindruck ausgehen, den das Verhalten einer Person auf uns ausübt. Ihre Handlungen dienen als Indizien ihres Charakters. Denn die Affekte, die die Grundlage der Handlungsmotivation darstellen, treten nicht willkürlich, isoliert und punktuell auf, sondern nach Maßgabe allgemeiner Gefühlsdispositionen, die, in einem bestimmten Mischungsverhältnis, den Charakter der Person prägen. Gegenstand der ethischen Bewertung sind diese Gefühlsdispositionen, die unter bestimmten Umständen im Verhalten einer Person symptomatisch werden (ebd., 3.2.1.1-4, S. 307).

Gefühlsreaktionen als Sensorium: Diese Bestimmung des Gegenstandes der moralischen Beurteilung ist jedoch nicht der wesentliche Grund, 
warum Humes Tugendethik als Gefühlstheorie der Moral zu bezeichnen ist. Entscheidend ist vielmehr, dass auch die ethischen Kriterien der Klassifikationen eines Charakters als tugend- oder lasterhaft in unseren Gefühlen liegen. Moral-sense-Theoretiker wie Hume begreifen unsere Gefühlsreaktionen als eine Art Sensorium: Werden wir Zeug/innen einer Handlung - ein Obdachloser hilft einer gestrandeten Autofahrerin; ein Präsidentschaftskandidat macht sich öffentlich über die Behinderung eines Journalisten lustig -, dann löst der Eindruck dieser Handlung, die bestimmten Affekten der handelnden Person entspringt und die in den von der Handlung unmittelbar betroffenen Personen ebenfalls bestimmte Affekte erzeugt, in uns als Beobachter/innen ebenfalls Affekte aus. Vermittelt durch unser natürliches Empathievermögen entsteht gewissermaßen eine Gefühlsresonanz. Deren Art und Intensität mag freilich davon abhängen, ob wir mit der wahrgenommenen Situation irgendwelche eigenen Interessen verbinden und inwieweit wir uns mit den beteiligten Personen verbunden fühlen oder identifizieren. Soweit unsere Gefühlsreaktionen in dieser Weise durch partikulare Interessen und persönliche Bindungen bestimmt sind, können sie kaum als Basis der moralischen Beurteilung verstanden werden.

Der unparteiische Beobachter: Wir können uns nun aber vorstellen, dass auch eine Person, die eine soziale Interaktion ganz unvoreingenommen betrachtet - die keinerlei besonderes Interesse mit der Handlung oder mit deren Konsequenzen verbindet und mit keiner der beteiligten Personen durch ein besonderes Verhältnis der Nähe oder Identifikation verbunden ist -, ebenfalls eine emotionale Resonanz in sich verspüren würde. Die Gefühle, die sie der handelnden Person aufgrund der wahrgenommenen äußeren Handlung zuschreibt, sind ihr aus eigener innerer Wahrnehmung grundsätzlich vertraut. Ebenso vertraut ist ihr die emotionale Wirkung der Handlung auf die von der Handlung betroffene Person. Diese grundsätzliche Vertrautheit erklärt sich aus der allgemeinen Ähnlichkeit der menschlichen Natur. Im Sinne einer generalisierten Einfühlung oder sympathie identifiziert sie sich mit allen beteiligten oder betroffenen Personen in derselben unparteiischen und unvoreingenommenen Weise. (Hume und andere Moral-sense-Theoretiker/innen verwenden das Wort "Sympathie» hier im Sinne dessen, was wir heute als Empathie bezeichnen würden: Sie meinen weniger eine positive Voreingenommenheit zugunsten einer anderen Person als vielmehr die Einfühlung in deren Zustand und Situation; vgl. ebd., 3.3.2.2-6, S. 378 ff.) Die Abwesenheit eigener Interessen oder Parteilichkeiten bedeutet also nicht, dass jene Person kalt und unbeteiligt wäre. Vielmehr nimmt sie sozusagen für alle Beteiligten gleichermaßen Partei. Sie vertritt, wie Hume formuliert, den common point of view (ebd., 3.3.1.30, S. 377). Nennen wir diese Person einen unparteiischen Beobachter (impartial spectator). Die Gefühlsreaktionen eines solchen unparteiischen Beobachters auf die Interaktionen zwischen einer handelnden Person (agent) und einem Handlungsbetroffenen (recipient) sind nach Auffassung der Moral-sense-Theoretiker das Kriterium der moralischen Beurteilung.

Realität des unparteiischen Beobachters: Der unparteiische Beobachter ist natürlich nicht ein konkretes Individuum, das wir aufgrund ihrer 
besonderen ethischen Autorität in Zweifelsfällen zu Rate ziehen müssten. Ebenso wie bei der Idee des hobbesschen oder rawlsschen Sozialvertrags handelt es sich beim unparteiischen Beobachter zunächst um ein fiktives Idealmodell (vgl. Kymlicka 1993). Allerdings ist der unparteiische Beobachter mehr als ein bloßes Gedankenexperiment. Denn wir können offenbar tatsächlich versuchen, gleichsam hinter unsere eigenen Interessen und Loyalitäten zurückzutreten, um uns zumindest annäherungsweise in die Rolle eines unparteiischen Beobachters zu versetzen. Mehr noch: Als Personen mit einem entwickelten moralischen Sinn haben wir die Rolle des unparteiischen Beobachters so internalisiert, dass ihre Stimme mehr oder weniger deutlich im Konzert unserer Gefühle vernehmbar ist. Zwar ist es mitunter schwierig, parteiliche und unparteiliche Gefühle auseinanderzuhalten. So ist es schwer, an jemandem, den man als Gegner betrachtet, moralische Tugenden zu bemerken. Aber so wie jemand mit feinem Gehör und hinreichender Selbstbeherrschung imstande ist, wahrzunehmen, dass die Stimme seines Gegners angenehm ist, sind wir prinzipiell imstande, unsere interessenlose moralische Billigung oder Missbilligung von unseren parteilichen Gefühlen zu unterscheiden (Hume 2007, 3.1.2.4, S. 304 f.). Es muss dafür kein künstliches Element in unseren Seelenhaushalt eingeführt werden. Denn die moralischen Gefühle der Billigung oder Missbilligung entstehen aufgrund der natürlichen `Sympathie`, die uns am Wohl anderer Personen Anteil nehmen lässt. Wir müssen lediglich die Verzerrungen und Einseitigkeiten in Rechnung stellen, die durch unsere eingeschränkte Perspektive und zu mal unsere Partikularinteressen zustande kommen.

Kategorisierung der Tugenden

Hume 2007, 3.3.3.9, S. 386

Sozial- und Individualtugenden: Charakterzüge, die Gegenstand der moralischen Billigung sind, teilen Hume zufolge zwei Merkmale: Sie sind entweder nützlich oder angenehm, und zwar sind sie nützlich oder angenehm entweder für den Handelnden selbst oder für andere Personen. Humes Tugendethik umfasst also Sozial- und Individualtugenden und steht insofern zwischen eudaimonistischen oder perfektionistischen Konzeptionen, die das Glück oder die Vollkommenheit des oder der Handelnden in den Mittelpunkt stellen, und jenen modernen Moral- und Tugendtheorien, die ausschließlich Verpflichtungen gegenüber anderen oder soziale Tugenden betrachten. Als »ultimativer Test« zur Beurteilung der Tugend einer Person kann Hume zufolge gelten, dass eine Person genau dann von vollkommener Tugend ist, wenn ich mir kein persönliches Verhältnis zu dieser Person vorstellen kann (einschließlich der Vorstellung, dass ich selbst diese Person bin), in dem ich mich nicht gerne befinden würde:

"And 'tis a most certain rule, that if there be no relation of life, in which I cou'd not wish to stand to a particular person, his character must so far be allow'd to be perfect. If he be as little wanting to himself as to others, his character is entirely perfect. This is the ultimate test of merit and virtue."

Darüber hinaus unterscheidet Hume im Treatise natürliche und künstliche Tugenden - eine von Zeitgenossen kontrovers diskutierte Innovation Humes im Vergleich zu den früheren Vertretern des Sentimentalis- 
mus. (In der zweiten Enquiry tritt die Unterscheidung in den Hintergrund, wird der Sache nach aber nicht aufgegeben.)

Natürliche Tugenden: Als natürliche Tugenden betrachtet Hume diejenigen Charakterzüge, deren Ausübung im Normalfall auch unabhängig von bestehenden sozialen Institutionen oder Konventionen unmittelbar angenehm oder nützlich für die Handelnden oder die von der Handlung Betroffenen sind (ebd., 3.3.1.28, S. 377). Dazu zählen soziale Tugenden der Güte und des Wohlwollens wie Großmut, Menschlichkeit, Mitleid, Dankbarkeit, Freundschaft, persönliche Treue, Engagement für andere, Uneigennützigkeit und Freigiebigkeit (ebd., 3.3.3.3, S. 385). Sie sind Ausdruck von unmittelbar angenehmen Gefühlen. Aufgrund des emotionalen Resonanzmechanismus, den Hume Sympathie nennt, sind diese Gefühle "so contagious, that they pass with the greatest facility from one person to another, and produce correspondent movements in all human breasts « (ebd., 3.3.3.5, S. 386). Sie erzeugen deshalb im unvoreingenommenen Betrachter ebenfalls ein unmittelbar positives Gefühl. Wie die Liebe unmittelbar angenehm ist, so ist der Hass unmittelbar unangenehm. Daher verurteilen wir alle Leidenschaften, die eine wesentliche Beimengung des Letzteren haben (ebd., 3.3.3.4, S. 385), am meisten die Grausamkeit, die das am meisten verachtete aller Laster darstellt (ebd., 3.3.3.8, S. 386). Zu den natürlichen Tugenden zählen auch Individualtugenden, die für tugendhafte Personen selbst angenehm oder nützlich sind, beispielsweise Fleiß (ebd., 3.3.1.13, S. 371), oder Tugenden der Seelengröße wie ein angemessenes Selbstbewusstsein, das von übertriebener Eitelkeit eben so weit entfernt ist wie von zerknirschter Selbsterniedrigung (ebd., 3.3.2, S. $378 \mathrm{ff}$.).

Künstliche Tugenden: Die Struktur der künstlichen Tugenden ist komplizierter. Diese Tugenden gewinnen ihre Nützlichkeit aus ihrem unverzichtbaren Beitrag zur Aufrechterhaltung gesellschaftlicher Institutionen, die das allgemeine Wohl der Gemeinschaft befördern. Entsprechend ist die genaue Beschaffenheit derjenigen Handlungen, die durch die künstlichen Tugenden motiviert werden, von der Ausgestaltung jener Institutionen abhängig. Der Inhalt der künstlichen Tugenden hängt deshalb zu einem gewissen Teil von der Struktur der jeweiligen Gesellschaft, ihrer Institutionen und Konventionen, ab. Auch die Struktur der emotionalen Reaktion auf die Wahrnehmung von Handlungen, die als Ausdruck der künstlichen Tugenden interpretiert werden können, ist komplizierter als in Bezug auf die natürlichen Tugenden. Das Gefühl der moralischen Billigung stellt sich in Bezug auf die künstlichen Tugenden nämlich nicht als unmittelbarer Widerhall derjenigen positiven Gefühle ein, durch die die Handlung motiviert war, oder die das unmittelbare Resultat der Handlung bei den von ihr Betroffenen sind. Das positive Gefühl, das Grundlage der moralischen Billigung ist, ist hier vielmehr das Ergebnis der empathischen Anteilnahme an dem allgemeinen gesellschaftlichen Wohl, das durch die künstlichen Tugenden ermöglicht wird. Es ist ein "general sense of common interest; which sense all the members of the society express to one another, and which induces them to regulate their conduct by certain rules« (ebd., 3.2.2.10, S. 315). Die Entwicklung der künstlichen Tugenden vollzieht sich parallel mit der Entwicklung der gesellschaftli- 
Kritische Anknüp-

fung an Hobbes

Hume 2007,

3.2.2.10, S. 315 chen Konventionen und Institutionen wie denen des Eigentums, des Rechts oder der Vertragstreue, die durch die Tugenden gestützt werden.

Konvention statt Vertrag: Die Respektierung des Eigentums anderer, die Einhaltung des Rechts und die Vertragstreue entstehen zunächst als bloße Konventionen. Aufgrund ihrer offenkundigen Nützlichkeit als Grundlagen fruchtbarer sozialer Kooperation gewinnen sie zunehmend an Stabilität und Verbindlichkeit. Einerseits folgt Hume damit Hobbes in der Auffassung, dass Institutionen wie Eigentum und Recht eine Grundlage in dem rationalen Eigeninteresse haben, das jeder Einzelne an den durch sie ermöglichten Vorzügen eines friedlichen Zusammenlebens und der kulturellen und wirtschaftlichen Kooperation nimmt. (Hingegen widerspricht er damit der Auffassung Lockes. Nach dessen Auffassung, die bis heute großen Einfluss auf liberale und libertaristische Konzepte politischer Philosophie ausübt, hat das Eigentumsrecht eine naturrechtliche Grundlage, die von sozialen Vereinbarungen unabhängig ist. Das Eigentumsrecht resultiert Locke zufolge aus dem uns von Gott verliehenen Recht an unserer eigenen Person und Arbeitskraft. Sobald wir einen zuvor herrschaftslosen Gegenstand in Besitz nehmen und durch eigene Arbeit mit unserer Person `mischen`, geht er in unser Eigentum über, sofern genug Gegenstände vergleichbarer Qualität zur Verfügung stehen; Locke 2016; vgl. Becker 1977, Kap. 4; Waldron 1988). Humes Interpretation der genannten Institutionen unterscheidet sich andererseits in zweierlei Weise aber auch von der hobbesschen. Gegen die vertragstheoretische Auffassung, wonach ein rechtlich geordnetes Gemeinwesen dadurch zustande kommt, dass Personen, die sich selbst noch in einem vorrechtlichen Naturzustand befinden, miteinander einen Vertrag schließen, wendet er ein, dass die Institution des Vertrages selbst nur vor dem Hintergrund bereits bestehender sozialer Konventionen einen verständlichen Sinn hat (vgl. das in Kap. 3 bereits angesprochene >Münchhausen-Problem hobbesscher Vertragstheorien). Humes Alternativvorschlag beruht in der Annahme, dass Institutionen wie die des Eigentums aus konventionellen Übereinkünften hervorgehen, die sich als vorteilhaft erweisen. Er illustriert dies anhand der stillschweigenden Übereinkunft zweier Ruderer, die in demselben Boot sitzen:

"l observe, that it will be for my interest to leave another in possession of his goods, provided he will act in the same manner with regard to me. He is sensible of a like interest in the regulation of his conduct. When this common sense of interest is mutually express'd, and is known to both, it produces a suitable resolution and behaviour. And this may properly enough be call'd a convention or agreement betwixt us, tho' without the interposition of a promise [...]. Two men, who pull the oars of a boat, do it by an agreement or convention, tho' they have never given promises to each other."

Egoismus und Altruismus: Ein zweiter Differenzpunkt liegt darin, dass Hume, anders als Hobbes, das rationale Eigeninteresse nicht als einzige psychologische Grundlage für die Entstehung und Aufrechterhaltung der Institutionen betrachtet. Zwar begreift er diese Institutionen, zumal die Rechtsordnung, explizit auch als Maßnahmen zur Kompensation der 
Grenzen des menschlichen Altruismus. Der emotionale Resonanzmechanismus der Sympathie funktioniert nämlich vor allem im nahen sozialen Umfeld. Für das Wohl und Wehe von Personen, die in größerer räumlicher oder zeitlicher Entfernung leben, ist er deutlich weniger empfindlich - die menschliche Großzügigkeit ist begrenzt (ebd., 3.2.2., S. 317; für eine aktuellere Diskussion solcher »altruism failures« vgl. Kitcher 2011). Zugleich ist die Ausdehnung von Kooperationszusammenhängen über das engere persönliche Umfeld hinaus aber von großem Vorteil. Insofern sind Institutionen und künstliche Tugenden, die egoistische Motive gewissermaßen in eine pro-soziale Richtung umlenken (ebd., 3.2.2.13, S.316), gegen die Schwächen unserer eigenen Natur gerichtet. Hume wehrt sich jedoch dagegen, den natürlichen Egoismus des Menschen zu übertreiben. Ohne das `Monster von Malmesbury`namentlich zu erwähnen, stellt er fest, dass die Beschreibungen der menschlichen Selbstsucht, "which certain philosophers delight so much to form of mankind « ebenso weit von der wahren Natur entfernt seien wie die Berichte von Monstern, denen wir in Fabeln und Dichtungen begegnen (ebd., 3.2.2.5, S. 313). Hume gelingt es damit, Zweifel an der Stabilität einer gesellschaftlichen Ordnung, die wesentlich auf Eigeninteresse gegründet ist, zumindest $\mathrm{zu}$ beschwichtigen. Denn Eigeninteresse ist zwar auch Hume zufolge das ursprüngliche Motiv für die Etablierung einer Rechtsordnung. Diese Ordnung wird jedoch gestützt durch eine Sympathie für das Allgemeinwohl, die sich in der moralischen Anerkennung der künstlichen, die soziale Ordnung tragenden Tugenden manifestiert (ebd., 3.2.2.24, S. 320 f.). Neben die Furcht vor Strafe tritt bei Hume also eine in allgemeinem Wohlwollen begründete bürgerliche Loyalität als Motiv für die Aufrechterhaltung der Rechtsordnung.

\section{3 | Diskussion}

Der schlaue Schurke: Ob Hume das Trittbrettfahrer-Problem damit vollständig gelöst hat, ist zweifelhaft. Hume thematisiert es vor allem in der moralphilosophischen Enquiry. Er diskutiert dort den Fall eines 'schlauen Schurken` (»sensible knave«) der, ähnlich wie der erwähnte `Narr in Hobbes' Leviathan, aus egoistischen Motiven gegen die Forderungen der Gerechtigkeit verstößt, sobald er überzeugt ist, dass ein einzelner Regelverstoß für ihn selbst großen Nutzen bringt, ohne die auch für ihn profitable Sozialordnung insgesamt zu gefährden (Hume 1998, 9.2.23 S. 81 f.). Hume äußert selbst Zweifel, inwieweit man diesem Schurken Gründe vermitteln kann, in solchen Fällen seinen Eigennutz zurückzustellen. Wenn die wesentliche motivationale Grundlage der Gerechtigkeitstugend umgelenktes Eigeninteresse ist, ist tatsächlich nicht leicht zu sehen, was man einem sschlauen Schurken entgegenhalten könnte. Die von Hume selbst vorgeschlagenen Antwortversuche sind nach Meinung der meisten Interpret/innen nicht völlig überzeugend (vgl. kritisch Baron 1982; Culp 2013; Gauthier 1992; Postema 1988; metakritisch Baier 1992; Frazer 2010, S.78f.). Die einschlägigen
Eigennutz und allgemeines Wohlwollen 
Selby-Bigge in Hume 1902, S. vii

Hume 2007, 3.1.2.10, S. 305
Kontroversen erklären sich zum großen Teil aus Unklarheiten bezüglich der Frage, wie genau die Motive von Akteuren bei der Entstehung der künstlichen Tugend zusammenwirken (s. u. und Darwall 1995, Kap. 10).

Spannungen in Humes praktischer Philosophie: Humes Moraltheorie besticht durch die Anschaulichkeit und Differenziertheit der in den Blick genommen ethischen Phänomene, ihren Gedankenreichtum, die Originalität, in der verschiedene Theorietraditionen vermittelt und weiterentwickelt werden, die Radikalität der philosophischen Grundannahmen und die zumal im Treatise äußerst dichten, trotz großen Detailreichtums klar strukturierten Darstellungen der einzelnen Argumentationsschritte. Der überragenden Bedeutung des humeschen Beitrags zur moralphilosophischen Tradition entspricht die Vielfalt der Diskussionen, die sie ausgelöst hat. Nicht wenige davon betreffen die Frage, wie die im Einzelnen jeweils als zwingend präsentierten Überlegungen Humes miteinander vereinbart werden können. Zwar übertreibt der Hume-Herausgeber SelbyBigge mit seiner häufig zitierten Behauptung, Hume sage

"so many different things in so many different ways and different connexions, and with so much indifference to what he has said before, that it is very hard to say positively that he taught, or did not teach, this or that particular doctrine."

Spannungsreich sind Humes Überlegungen aber durchaus.

Anti-Naturalismus oder Naturalismus: So argumentiert Hume in vielen Passagen als vehementer Kritiker des moralphilosophischen Naturalismus. Am Ende des ersten Teils von Buch 3 des Treatise geht er die Frage, ob Moral aus der Natur stammen kann, systematisch an. Im ersten Schritt unterscheidet er mit großer Umsicht unterschiedliche Interpretationen des Begriffs »Natur«. Im zweiten Schritt argumentiert er, dass die These, die Tugend bezeichne das Natürliche, unter jeder dieser Interpretationen unhaltbar sei. Verstehe man das Natürliche als Gegenteil des Wunderbaren, seien Tugend und Laster gleichermaßen natürlich. Verstehe man das Natürliche als Gegenteil des Ungewöhnlichen, dann sei Tugend vielleicht das am allerwenigsten Natürlichste. Begreife man das Natürliche als Gegenteil des Künstlichen, so stünden Tugend und Laster erneut genau auf derselben Stufe. Es sei daher schlechthin unmöglich, dass die Unterschiede zwischen dem Natürlichen und dem Unnatürlichen in irgendeiner Weise die Unterschiede zwischen Tugend und Laster markierten:

"'Tis is impossible, therefore, that the character of natural and unnatural can ever, in any sense, mark the boundaries of vice and virtue."

Das ist eine starke und eindeutige Aussage. Hume unterstreicht sie noch durch die Behauptung, nichts könne sunphilosophischer sein als jene ethischen Systeme, die die Tugend mit dem Natürlichen und das Laster mit dem Unnatürlichen gleichsetzen (ebd.). Nur wenig später behauptet Hume jedoch überraschenderweise, dass wir uns stets an der "natürlichen« und »üblichen« Stärke der Leidenschaften orientieren, wenn wir Tugend und Laster beurteilen (»we always consider the natural and usual 
force of the passions, when we determine concerning vice and virtue«; ebd., 3.2.1.18, S. 311; Hervorh. im Orig.) - ganz so, wie wir die Schönheit eines Körpers am Standard der arttypischen Proportionen bemessen (ebd.). Wenn die Leidenschaften in die eine oder andere Richtung sehr stark von dem allgemeinen Maß abwichen, würden sie stets als lasterhaft verurteilt. Seine Überlegungen, die einer Aristoteles-Paraphrase verblüffend nahe kommen, schließt Hume mit den Worten:

"Our sense of duty always follows the common and natural course of our passions."

Hume 2007, 3.2.1.18, S. 311

Es ist kaum zu sehen, wie diese Stellungnahmen miteinander vereinbart werden könnten. Aus den jeweiligen Kontexten wird klar, dass Hume nicht nur die Auffassungen anderer Autoren wiedergeben, sondern seine eigene Position ausdrücken möchte. Auch besteht das Problem unabhängig davon, ob man Hume als einen Vertreter des Nonkognitivismus, etwa des Emotivismus, interpretiert, oder ob man ihm eine kognitivistische, beispielsweise subjektivistische oder irrtumstheoretische Position zuschreibt (zu diesen metaethischen Positionen siehe Kap. 8). Die Spannung zwischen den beiden zitierten Passagen bleibt unter jeder Interpretation bestehen, die Konsistenzforderungen wenigstens auf der Ebene der ethischen Theorie erhebt (selbst wenn sie dies, wie der klassische Emotivismus, auf der `Objektebene der moralischen Äußerungen selbst nicht tut): Entweder ist es "unmöglich", dass die Unterscheidung zwischen dem Natürlichen und Unnatürlichen »in irgendeinem Sinne« den Unterschied zwischen Tugend und Laster bezeichnet, oder unser moralischer Sinn "folgt immer « dem normalen und natürlichen Lauf der Leidenschaften und deren natürlicher und normaler Stärke und Proportion.

Universalismus oder Partikularismus: Eindeutig ist Humes Position allerdings in der Aussage, dass moralische Qualitäten nicht an objektiv beschreibbaren Merkmalen des äußeren Verhaltens haften können (s. o., die Diskussion des Mord-Beispiels). Moralische Qualitäten schreiben wir nur nach Maßgabe der subjektiven Gefühlsresonanz zu, die ein beobachtbares Verhalten bei unparteilicher Betrachtung in uns auslöst. Die moralisch maßgebliche Gefühlsresonanz bemisst sich aber ihrerseits anscheinend an natürlichen und objektiven Standards - »the natural and usual force of the passions « (s. o.). Mehr noch: Anscheinend soll sie sich auch daran orientieren. Hier wird eine weitere Spannung deutlich, nämlich eine Spannung zwischen Universalismus und Partikularismus: Einerseits müssen wir, um den »common point of view« einzunehmen, den natürlichen Partikularismus unserer Privatperspektive hinter uns lassen; unsere Sympathie soll für alle Beteiligten und Betroffenen gleichermaßen sensibel sein. Andererseits sollen wir auch in der Rolle eines unparteiischen Beobachters unsere moralischen Urteile anscheinend noch an den von der Natur vorgegebenen Abstufungen des Wohlwollens oder der Solidarität orientieren:

"A man naturally loves his children better than his cousins, his cousins better than strangers, where every thing else is equal. Hence arise our common measures of duty, in preferring the one to the other."
Hume 2007,

3.2.1.18, S. 311 
Anatom oder Deskriptive oder normative Ethik: Wie ist der hier vollzogene Übergang Maler? von den Standards des Normalen und Natürlichen zu Maßstäben der Tugend zu rechtfertigen - zumal vor dem Hintergrund von Humes eigenen Ausführungen zum Sein-Sollens-Fehlschluss? Warum sollen wir uns an der `normalen` und `natürlichen`Stärke der verschiedenen Leidenschaften orientieren? Eine denkbare Reaktion könnte in der Annahme liegen, dass Hume im Grunde gar keine normative, sondern nur eine deskriptive Ethik entwickeln will - dass er lediglich beschreiben und erklären will, wie und warum wir faktisch Tugendurteile fällen, aber nicht das Anliegen verfolgt, diese Urteile auch zu rechtfertigen. Tatsächlich haben viele Aussagen Humes über die Zuschreibung von Tugend und Laster den Charakter von Beschreibungen, die wiedergeben, auf welcher Grundlage moralische Bewertungen üblicherweise vorgenommen werden, aber nicht begründen, warum sie auf diese Weise vorgenommen werden sollten. In einem Brief an Hutcheson (Hume 1932, Bd. 1, S. 32 f.) und später in einer Passage der Enquiry Concerning Human Understanding (Hume 2000, 1.1.8, S. 8) erläutert Hume denn auch, dass er sich primär als Anatom der Moral versteht, der gleichsam Struktur und Funktion der Knochen, Muskeln und Organe unseres Gefühlslebens analysiert, und nicht als Maler, der das Bild der Tugend im Hinblick auf die Wirkung auf den Betrachter komponiert, bei dem er starke Gefühle auslösen will. In vielen anderen Passagen macht Hume aber wiederum deutlich, dass er die Moralphilosophie für eine praktische Disziplin hält, die überhaupt nur dadurch Berechtigung erlangt, dass sie auf das Handeln der Menschen Einfluss ausübt (Hume 2007, 3.1.1.1, S. 293; 3.1.1.5, S. 294; vgl. auch Hume 1998, 1.7, S. 5).

Pflichtgefühl und das Gute an sich: Komplikationen ergeben sich in diesem Kontext durch Humes Äußerungen zur Frage, ob die Handlungen einer Person durch Vorstellungen von Pflicht und Gerechtigkeit motiviert sein können. Hume scheint dies notwendigerweise annehmen zu müssen, wenn er an der These festhalten will, dass ethische Theorien motivierenden Einfluss haben, und er sagt es auch explizit:

Hume 2007, 3.1.1.5, S. 294

Pflicht als Motiv?
"[C]ommon experience [...] informs us, that men are often govern'd by their duties, and are deter'd from some actions by the opinion of injustice, and impell'd to others by that of obligation."

Im Zusammenhang mit der Einführung der künstlichen Tugenden vertritt Hume dann jedoch die Auffassung, dass tugendhafte Handlungen niemals durch das Bewusstsein ihrer Tugendhaftigkeit motiviert sein könnten. Er argumentiert wie folgt: Um zu beurteilen, ob eine Handlung tugendhaft ist, müssen wir ihr Motiv beurteilen. Das können wir nur, wenn bereits ein vorgängiges Motiv vorliegt, das wir im Hinblick auf seine Tugendhaftigkeit beurteilen können. Die Tugendhaftigkeit der Handlung kann daher nicht selbst erstes Handlungsmotiv sein, denn anderenfalls gerieten wir in einen Zirkel, in dem die Grundlage der Prüfung zugleich ihr Ergebnis ist und umgekehrt (ebd., 3.2.1.2-7, S. 307 f.). Es sei daher 
"establish'd as an undoubted maxim, that no action can be virtuous, or morally good, unless there be in human nature some motive to produce it, distinct from the sense of its morality."

Wie Abramson (2002, S. 304) ausführt, ist diese Auffassung jedoch mit Humes Internalismus schwer zu vereinbaren.

Handlungs- und Rationalitätstheorie: Wie Moralphilosophie unter den humeschen Annahmen Handlungen motivieren können soll, ist freilich auch aufgrund seiner Handlungs- und Rationalitätstheorie nicht leicht zu verstehen. Wenn Vernunft nur als Sklavin der Leidenschaften dient, stellt sich die Frage, inwieweit moralische Überzeugungen und ethische Theorien überhaupt die Richtung unserer Motivation beeinflussen können. Falls es sich bei moralischen Überzeugungen um sechter Überzeugungen handelt - das heißt, wenn Sie im Sinne des Kognitivismus den Charakter von wahrheitsfähigen beliefs haben - und falls es überdies stimmt, dass beliefs allein niemals Handlungen motivieren können, dann muss anscheinend die internalistische Annahme aufgegeben werden, wonach moralische Überzeugungen notwendigerweise motivierende Kraft haben (und nicht nur dann, wenn zufälligerweise ein passendes desire hinzutritt). Eine emotivistische Deutung hingegen, wonach wir moralische Urteile gar nicht als >echte` Urteile - das heißt: nicht als Ausdruck wahrheitsfähiger Überzeugungen, sondern Ausdruck von desires - verstehen müssen, könnte zwar im Rahmen des Belief-desire-Modells erklären, warum diese (Pseudo-)Urteile notwendig an motivationale Dispositionen (eben die ausgedrückten desires) gebunden sind. Sie könnte aber wiederum kaum erklären, wie ethische Argumentation möglich ist oder wie moralphilosophische Theorien die moralischen Haltungen von Rezipient/ innen auf rationale Weise beeinflussen könnten (einen entsprechenden Versuch unternimmt etwa Gibbard 1990; dazu kritisch Habermas 1996, S. 28 ff.; Sinnott-Armstrong 1993). Wie Korsgaard bemerkt, lässt die Unterscheidung zwischen Anatom und Maler gar keinen Raum für die eigentliche normative Frage: Der Anatom beschreibt und erklärt nüchtern das Zustandekommen von Gefühlen, der Maler sucht sie anzufachen. Inwieweit die Gefühle gerechtfertigt werden können, fragt jedoch keiner von beiden (Korsgaard 1996, S. 52).

Erklärende oder normative Gründe: Folgt die Schwierigkeit, in Humes Theorie Raum für die Rechtfertigung (nicht nur Erklärung) von Haltungen, Entscheidungen oder ethischen Urteilen zu finden, tatsächlich unmittelbar aus seiner Handlungs- und Rationalitätstheorie? Man könnte einwenden, dass die Rolle, die er der Vernunft zubilligt, bedeutender ist, als seine zugespitzten Beispiele suggerieren. Denn auch eine instrumentelle Vernunft, die uns lediglich auf Objekte unserer Verlangen und auf Mittel zu deren Befriedigung hinweist, müsse diese Verlangen faktisch in bedeutender Weise formen. Dies gelte zumal dann, wenn es sich um Verlangen sehr allgemeiner Art handele. Wie schon erwähnt, nimmt Hume ja sogar die Existenz eines "general appetite to good, and aversion to evil, consider'd merely as such" an (Hume 2007, 2.3.4.8, S. 268). Allerdings entwickelt Hume keine Konzeption einer Vernunft, die uns die Abwägung zwischen verschiedenen Objekten und/oder Wegen zur Befriedigung
Hume 2007,

3.2.1.7, S. 308, Hervorh. im Orig.

Problematische Rolle der praktischen Vernunft 
Kein Raum für normative Gründe (und damit zugleich eine Präzisierung) derart allgemeiner Verlangen ermöglichen würde. Im Gegenteil: Zwar räumt er gelegentlich eine mögliche Differenz zwischen dem faktisch Erstrebten (dem Objekt unserer stärksten Begierden) und dem (aus unserer eigenen Perspektive) Erstrebenswerten ein. Statt an diese Differenz jedoch normative Überlegungen anzuknüpfen, sucht er sie sogleich durch eine mechanistische Psychologie wieder aus der Welt zu schaffen, die nach dem Modell der Hebelwirkung erklären soll, wieso schwächere Affekte sich manchmal gegen stärkere durchzusetzen vermögen (ebd., 2.3.3.6, S. 267). Vieles spricht für Michael Smiths Auffassung, wonach es Hume nicht gelungen ist, das Wesen normativer Gründe (im Unterschied zu motivationalen Gründen) verständlich zu machen (Smith 1994, insbes. Kap. 4 und 5). Wenn wir uns in deliberativer Perspektive, d. h. aus der Perspektive des Akteurs im Zuge der Handlungsorientierung, fragen, was wirklich erstrebenswert ist, scheinen wir (häufig) nicht einfach >in unsere eigene Brust zu blicken und $\mathrm{zu}$ fragen, welches unserer bereits vorliegenden Verlangen das stärkste ist. Eine alternative Theorie normativer Gründe stellt Hume jedoch nicht zur Verfügung.

Ausblick: Hume begründet seine Kritik des ethischen Rationalismus mit einer Theorie der Vernunft, die in ihr nur eine Sklavin a-rationaler Verlangen sieht. Er wendet sich vehement gegen einen ethischen Naturalismus, der das Tugendhafte mit dem Natürlichen identifizieren will. Unklar bleibt allerdings, inwieweit die von ihm vertretene Gefühlsethik selbst eine Form des Naturalismus verkörpert. Charakteristisch für Humes Version des Sentimentalismus ist die Unterscheidung zwischen natürlichen und künstlichen Tugenden. Erstere sind unmittelbarer Ausdruck pro-sozialer Gefühle. Letztere erhalten ihren Inhalt erst durch soziale Vereinbarungen und ihre motivationale Kraft wesentlich durch umgelenktes Eigeninteresse, das allerdings durch ein generalisiertes Wohlwollen unterstützt wird. Humes immenser Einfluss lässt sich an den Inhaltsverzeichnissen aktueller Ethik-Einführungen nur bedingt ablesen. Der Humeanismus wird eher selten (z. B. in Beauchamp 2001) als eine der Hauptströmungen der Ethik aufgeführt. Mitunter werden überhaupt nur Utilitarismus und Kantianismus als allgemeine Schulen präsentiert; häufiger werden noch aristotelisch-tugendethische und kontraktualistische Ansätze genannt. Der Rolle Humes wird das auch deshalb nicht gerecht, weil Utilitarismus wie Kantianismus ohne den Einfluss Humes gar nicht denkbar wären: Kant begreift seine kritische Philosophie als direkten Gegenentwurf zu dem von Hume verteidigten Sentimentalismus (den Kant zunächst auch selbst vertreten hatte); und Bentham sieht den Utilitarismus in der direkten Tradition Humes. Ferner ist Hume auch eine zentrale Bezugsperson für Vertreter/innen evolutionstheoretischer Ansätze in der Ethik (Kitcher 2011) und nicht zuletzt auch für die Mitleidsethik und die jüngere Sorgeethik (vgl. Baier 1993). 


\section{Einführende Literatur}

Frazer, Michael L.: The Enlightenment of Sympathy: Justice and the Moral Sentiments in the Eighteenth Century and Today. Oxford/New York 2010.

Gräfrath, Bernd: Moral Sense und praktische Vernunft: David Humes Ethik und Rechtsphilosophie. Stuttgart 1991.

Mackie, John L.: Hume's Moral Theory. London/New York 1980.

Streminger, Gerhard: David Hume: Sein Leben und sein Werk. Paderborn 1995.

\section{Zitierte und weiterführende Literatur}

Abramson, Kate: »Two Portraits of the Humean Moral Agent«. In: Pacific Philosophical Quarterly 83 (2002), S. 301-334.

Anscombe, Elizabeth M.: Intention. Cambridge/London 1957.

Anscombe, Elizabeth M.: »Modern Moral Philosophy«. In: Philosophy 33 (1958), S. $1-19$.

Audi, Robert: Practical Reasoning and Ethical Decision. London 2006.

Baier, Annette C.: »Artificial Virtues and the Equally Sensible Non-Knaves«. In: Hume Studies 18/2 (1992), S. 429-439.

Baier, Annette C.: »Hume, der Moraltheoretiker der Frauen?«. In: Nagl-Docekal, Herta/Pauer-Studer, Herlinde (Hrsg.): Jenseits der Geschlechtermoral: Beiträge zur feministischen Ethik. Frankfurt a. M. 1993, S. 105-134.

Baron, Marcia W.: "Hume's Noble Lie: An Account of His Artificial Virtues«. In: Canadian Journal of Philosophy 12/3 (1982), S. 539-555.

Beauchamp, Tom L.: Philosophical Ethics: An Introduction to Moral Philosophy. Boston ${ }^{3} 2001$.

Becker, Lawrence C: Property Rights: Philosophic Foundations. London 1977.

Blackburn, Simon: Ruling Passions a Theory of Practical Reasoning. Oxford 1998.

Copp, David/Sobel, David: »Against Direction of Fit Accounts of Belief and Desire«. In: Analysis 61 (2001), S. 44-53.

Culp, Jonathan: »Justice, Happiness, and the Sensible Knave: Hume's Incomplete Defense of the Just Life«. In: The Review of Politics 75/2 (2013), S. 193-219.

Darwall, Stephen L.: The British Moralists and the Internal »Ought«: 1640-1740. Cambridge/New York 1995.

Davidson, Donald: Essays on Actions and Events. Oxford ${ }^{2} 2001$.

Foot, Philippa: Natural Goodness. Oxford 2001.

Gauthier, David: »Artificial Virtues and the Sensible Knave«. In: Hume Studies $18 / 2$ (1992), S. 401-427.

Gibbard, Allan: Wise Choices, Apt Feelings: A Theory of Normative Judgment. Harvard 1990.

Habermas, Jürgen: Die Einbeziehung des Anderen: Studien zur politischen Philosophie. Frankfurt a. M. 1996.

Hume, David: A Treatise of Human Nature, edited by David Fate Norton and Mary J. Norton. Clarendon Hume Edition Series, Bd. 1: Texts. Oxford/New York 2007.

Hume, David: An Enquiry Concerning the Principles of Morals: A Critical Edition. Hrsg. von Tom L. Beauchamp. Oxford/New York 1998.

Hume, David: An Enquiry Concerning Human Understanding. Hrsg. von Tom L. Beauchamp. Oxford/New York 2000.

Hume, David: Enquiries Concerning the Human Understanding and Concerning the Principles of Morals. Hrsg. von L. A. Selby-Bigge. Oxford 1902.

Hume, David: The Letters of David Hume. Hrsg. von J. Y. T. Greig. Oxford/New York 1932.

Jacobson-Horowitz, Hilla: »Motivational Cognitivism and the Argument from Direction of Fit«. In: Philosophical Studies 127/3 (2006), S. 561.

Kemmerling, Andreas: »Locke über die Wahrnehmung sekundärer Qualitäten«. In: Perler, Dominik/Wild, Markus (Hrsg.): Sehen und Begreifen: Wahrnehmungstheorien in der frühen Neuzeit. Berlin 2007, S. 203-233. 
Kitc Postema, Gerald J.: »Hume's Reply to the Sensible Knave«. In: History of Philosophy Quarterly 5/1 (1988), S. 23-40.

Kitcher, Philip: The Ethical Project. Cambridge/London 2011.

Korsgaard, Christine M.: The Sources of Normativity. Cambridge 1996.

Kymlicka, Will: »The Social Contract Tradition«. In: Singer, Peter (Hrsg.): A Companion to Ethics. Oxford 1993, S. 186-196.

Locke, John: Second Treatise of Government and A Letter Concerning Toleration [1714]. Oxford/New York 2016.

Railton, Peter: »Humean Theory of Practical Rationality«. In: Copp, David (Hrsg.): The Oxford Handbook of Ethical Theory. Oxford 2006, S. 265-281.

Ross, David: The Right and the Good [1930]. Oxford 2009.

Sinnott-Armstrong, Walter: "Some problems for Gibbard's norm-expressivism». In: Philosophical Studies 69/2 (1993), S. 297-313.

Smith, Michael A.: The Moral Problem. Oxford/Cambridge 1994.

Swanton, Christine: „Can Hume Be Read as a Virtue Ethicist?« In: Hume Studies 33/1 (2007), S. 91-113.

Waldron, Jeremy: The Right to Private Property. Oxford 1988.

Open Access Dieses Kapitel wird unter der Creative Commons Namensnennung 4.0 International Lizenz (http://creativecommons.org/licenses/by/4.0/deed.de) veröffentlicht, welche die Nutzung, Vervielfältigung, Bearbeitung, Verbreitung und Wiedergabe in jeglichem Medium und Format erlaubt, sofern Sie den/die ursprünglichen Autor(en) und die Quelle ordnungsgemäß nennen, einen Link zur Creative Commons Lizenz beifügen und angeben, ob Änderungen vorgenommen wurden.

Die in diesem Kapitel enthaltenen Bilder und sonstiges Drittmaterial unterliegen ebenfalls der genannten Creative Commons Lizenz, sofern sich aus der Abbildungslegende nichts anderes ergibt. Sofern das betreffende Material nicht unter der genannten Creative Commons Lizenz steht und die betreffende Handlung nicht nach gesetzlichen Vorschriften erlaubt ist, ist für die oben aufgeführten Weiterverwendungen des Materials die Einwilligung des jeweiligen Rechteinhabers einzuholen. 